
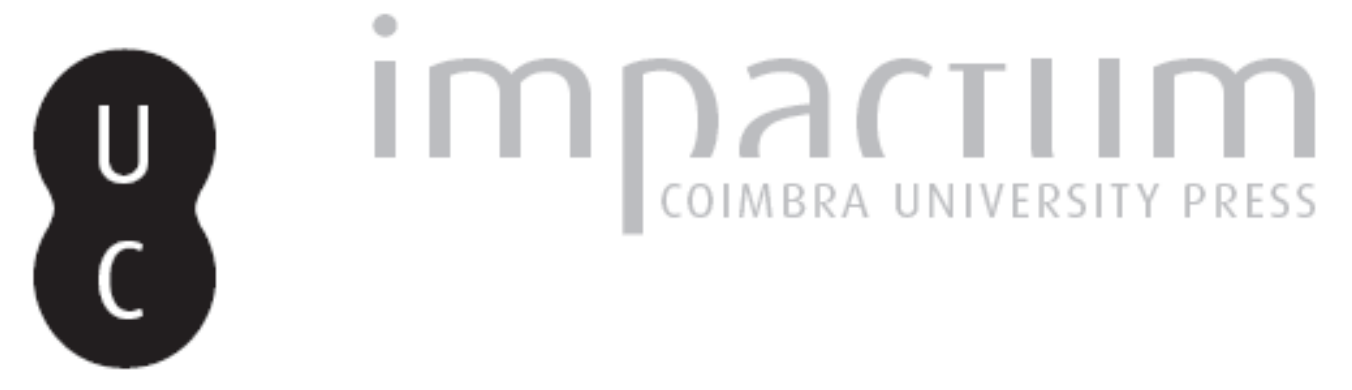

\title{
Plutarque et la question du caractère: le cas d' Antoine
}

\author{
Autor(es): Billault, Alain
}

Publicado por: International Plutarch Society

URL persistente:

URI:http://hdl.handle.net/10316.2/37606

DOI:

DOI:http://dx.doi.org/10.14195/0258-655X_3_2

Accessed : $\quad$ 26-Apr-2023 05:26:04

A navegação consulta e descarregamento dos títulos inseridos nas Bibliotecas Digitais UC Digitalis, UC Pombalina e UC Impactum, pressupõem a aceitação plena e sem reservas dos Termos e Condições de Uso destas Bibliotecas Digitais, disponíveis em https://digitalis.uc.pt/pt-pt/termos.

Conforme exposto nos referidos Termos e Condições de Uso, o descarregamento de títulos de acesso restrito requer uma licença válida de autorização devendo o utilizador aceder ao(s) documento(s) a partir de um endereço de IP da instituição detentora da supramencionada licença.

Ao utilizador é apenas permitido o descarregamento para uso pessoal, pelo que o emprego do(s) título(s) descarregado(s) para outro fim, designadamente comercial, carece de autorização do respetivo autor ou editor da obra.

Na medida em que todas as obras da UC Digitalis se encontram protegidas pelo Código do Direito de Autor e Direitos Conexos e demais legislação aplicável, toda a cópia, parcial ou total, deste documento, nos casos em que é legalmente admitida, deverá conter ou fazer-se acompanhar por este aviso.

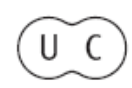




\section{PLOUTARCHOS, n.s.}

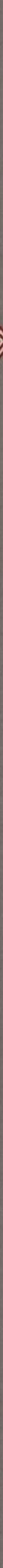

UNIVERSITY OF MÁLAGA (SPAIN)

UtAH STATE UNIVERSity, LOGAN, UTAH (U.S.A.) 


\title{
Plutarque et la question du caractère: le cas d'Antoine \\ Université de Paris-Sorbonne
}

\begin{abstract}
Plutarch writes Lives to portray characters. But some characters prove difficult to draw. In his Life of Antony, Plutarch portrays Antony as a man prone to disguise himself and living under many influences. His character appears to be elusive to such an extent that one may even wonder if it actually exists. The same question arises in How to tell a flatterer from a friend where Plutarch also comments on Antony. He considered him a special case and paints his character as an enigma.
\end{abstract}

Plutarque écrit des Vies pour repré senter des caractères. Il l'indique claire ment au début de la Vie d'Alexandre pour justifier sa décision de ne pas rela ter en détail toutes les actions du Macédonien et celles de César qu'il lui associe :

En effet, ce ne sont pas des his toires que nous écrivons, mais des vies et ce n'est pas dans les actions les plus éclatantes que se montre dans tous les cas la vertu ou le vice, mais souvent un petit fait, une paro le, une plaisanterie révèlent un ca ractère plus que des combats où tombent des milliers d'hommes, les batailles rangées et les sièges de villes les plus importants. De même, donc, que les peintres s'efforcent de saisir les ressemblances d'après le visage et les traits extérieurs qui font voir le caractère en se souciant très peu des autres parties du corps, de même, qu'on nous autorise à pénétrer davantage dans les signes distinctifs de l'âme et à nous en ser vir pour représenter la vie de chacun de ces deux hommes en laissant à d'autres les grands événements et les affrontements $(1,2-3)$.

Le choix du caractère comme sujet des Vies conduit donc Plutarque à trier la renseignements qui se trouvent à sa disposition et à en retenir d'abord ceux qui sont de nature à le révéler.En assem blant ces éléments, il composera une image (єіठолоıєı) de la vie des personnages qui l'intéressent. Il exprime la même intention au début de la Vie de Nicias (1) où il déclare avoir négligé les renseignements historiques déjà donnés par Thucydide au profit d'autres infor- 
mations moins connues, mais révélatri ces de la nature et du caractère de son personnage. Plutarque a donc une idée précise de ce qu'il fait. Cette idée implique que le caractère d'un homme est une réalité qui se laisse clairement percevoir à travers un certain nombre de signes. Plutarque les reconnaît, les inter prète et s'en sert pour composer la Vie de son personnage, une Vie qui consistera en une image de son caractère. Elle lui ressemblera comme un tableau réussi ressemble à son modèle. Mais le carac tère des hommes est-il toujours un mo dèle facile à percevoir ? La lecture de Plutarque permet d'en douter. $\mathrm{Si}$ sa conception des Vies est d'une clarté apollinienne, les caractères qu'elles met tent en scène ne se laissent pas toujours saisir aisément et Plutarque ne fait rien, bien au contraire, pour dissimuler cette difficulté. La Vie d'Antoine en donne sans doute l'exemple le plus frappant. Dans l'édition qu'il lui a consacrée, C. B. R. Pelling a bien montré que ce texte suit un mouvement d'approfondissement psy chologique à mesure que Plutarque nuan ce son récit et ses appréciations pour mieux rendre compte de la complexité de son personnage ${ }^{1}$. Celui-ci apparaît, en fait, constamment déchiré entre ses qualités (son courage, sa capacité à commander une armée, son désir de bien faire, sa générosité, son éloquence) et ses défauts (son goût pour les plaisirs et pour la débauche, sa grossièreté, sa simplicité d'esprit souvent proche de l'aveuglement, sa faiblesse de caractère). En soulignant ces déchirements, Plutarque brosse de son personnage un portrait en clair-obscur aussi impressionnant qu'énigmatique. En effet, les contradictions d'Antoine sont telles qu' on peut se demander s'il a vrai ment un caractère. Il est vrai que Plutarque a peint ce caractère, mais il a aussi mutiplié les traits qui invitent à s'in terroger sur son existence, qu'il s'agisse de la propension d'Antoine à changer de costume, des influences successives qu'il subit ou du rôle néfaste des flatteurs qui l'entourent. Ce rôle, Plutarque l'évoque aussi dans le traité Comment peut-on dis tinguer le flatteur de l'ami. Et le rappro chement des deux textes donne à penser que le caractère d'Antoine constituait bien un cas particulier aux yeux de Plutarque.

Dans la Vie d'Antoine, Plutarque re late cinq épisodes où son personnage se déguise. Lors du débat qui précède le début de la guerre civile entre César et Pompée, Antoine ne parvient pas à faire accepter par le sénat des propositions de César qui auraient peut-être permis d'é viter l'affrontement. Il est chassé du sénat par le consul Lentulus et se dégui se en esclave pour rejoindre César en compagnie de Quintus Cassius (5, 5-10). Plus tard, parti en Espagne pour rejoind re César, il revient soudain à Rome où le bruit court que César est mort et que les partisans de Pompée marchent sur la ville. Voulant faire une surprise à sa 
femme Fulvie, il arrive dans leur maison déguisé en esclave et se présente comme porteur d'une lettre d'Antoine. Comme Fulvie commence à la lire, il l'enlace et se fait reconnaitre (10.7-10). Lorsque César est assassiné, Antoine se déguise de nouveau en esclave pour se cacher puis, voyant que les conjurés ne mena cent personne, il entre en contact avec eux et reprend son activité politique (14.1-2). Plus tard, il essaye de se conci lier les troupes de Lépide en allant à la porte de son camp, avec des cheveux hirsutes, une barbe épaisse et un vête ment sombre pour les apitoyer, ce qu'il parvient à faire malgré Lépide (18). Il prend aussi un habit de valet pour par courir, la nuit, avec Cléopâtre déguisée en servante, les rues d'Alexandrie et lan cer des moqueries aux habitants qui répliquent par d'autres moqueries et même par des coups (29.2-3). Certains de ces épisodes ont suscité les scepticis me des commentateurs : le second a sans doute eu lieu avant, et non après la vic toire de César à Munda comme l'écrit Plutarque $^{2}$. Quant au troisième, il paraît à certains difficile à croire ${ }^{3}$. Mais Plutarque les relate tous sans formuler aucune réserve. Ils constituent, dans la Vie d'Antoine, une série dont la signifi cation mérite d'être recherchée en soi, indépendamment de leur exactitude his torique. On pourrait d'ailleurs ajouter à cette série un sixième épisode, avorté celui-là : pendant la campagne contre les Parthes, pour haranguer ses soldats, Antoine veut mettre un vêtement somb re afin d'exciter exciter leur pitié, mais ses amis l'en dissuadent et il revêt sa tenue pourpre de général pour se présen ter devant les troupes (44.3). Tous ces épisodes ne sont pas semblables. Avec Fulvie et Cléopâtre, Antoine se déguise pour s'amuser. Après son expulsion du sénat comme après l'assassinat de César, il le fait pour se cacher. Devant les trou pes de Lépide, son déguisement vise à susciter l'émotion. C'est un stratagème qu'il veut répéter pendant la guerre cont re les Parthes. Cette diversité d'inten tions autorise des interprétations diffé rentes. Dans une certaine mesure, ces épisodes apparentent la Vie d'Antoine à un récit d'aventures ${ }^{4}$. On peut y voir aussi une illustration de la capacité d'Antoine à s'adapter à toutes les situa tions, qu'il s'agisse de s'amuser, de se protéger du danger ou de tirer parti des apparences. D'autres personnages de Plutarque montrent une capacité analo gue en se déguisant : ainsi Alcibiade arrivé à Sparte après avoir abandonné l'expédition athénienne qu'il comman dait en Sicile adopte-t-il le mode de vie, le régime alimentaire et les habitudes vestimentaires des Lacédémoniens. Plu tarque souligne à cette occasion son ap titude à se conformer aux moeurs des peuples qu'il fréquente. Il la juge excep- 
tionnelle et la compare à la faculté mimétique du caméléon ${ }^{5}$. D'autre part, il commente en termes politiques le chan gement de costume d'Alexandre lancé à la conquête de l'Orient $^{6}$ : après qu'il a pénétré en Parthie, le conquérant se met à s'habiller comme un barbare sans adopter, toutefois, les larges pantalons, la robe ni la tiare des Mèdes. Il se com pose une tenue intermédiaire entre celle des Perses et celle des Mèdes. Elle est moins fastueuse que la seconde, mais plus majestueuse que la première. Plutarque juge cette combinaison adroi te et en donne deux explications : Alexandre voulait apprivoiser les barba res en s'habillant comme eux, ou habi tuer les Macédoniens à des coutumes nouvelles afin de les obliger plus tard à se prosterner devant lui. Plutarque ne considère donc pas comme secondaire la façon dont ses personnages s'habillent. Il y voit un signe de leurs intentions et de leur personnalité. Considéré dans cette perspective, le cas d'Antoine paraît très différent de ceux d'Alcibiade et d'Alexandre. Antoine ne se déguise pas pour réaliser un plan à long terme, mais pour répondre sur le moment aux cir constances qui s'imposent à lui. Il ne les évalue pas toujours avec exactitude : après la mort de César, il s'habille en esclave pour rien. Ses plans pour émou voir les troupes en portant une tenue sombre relèvent de calculs à courte vue. On le voit bien pendant la campagne contre les Parthes où il renonce à son idée lorsque ses amis s'y opposent. Enfin, dans quatre épisodes sur six, il se déguise en esclave, acceptant une déchéance apparente et provisoire, mais qui laisse à penser qu'il fait peu de cas de sa dignité. Cette indifférence aux signes extérieurs du rang social, ce déta chement vis-à-vis des apparences pour rait être considéré comme une preuve de simplicité et même comme une vertu philosophique, mais Plutarque ne le qua lifie pas ainsi. Il ne le commente pas et laisse ainsi la voie ouverte à une autre interprétation : au lieu de conduire sa vie et d'y demeurer en permanence sur le devant de la scène, Antoine est un per sonnage à éclipses qui disparaît souvent en se déguisant pour jouer un rôle. Les Alexandrins ne s'y trompent d'ailleurs pas. Ils prennent bien les bouffonneries d'Antoine, “disant qu'il portait le masque tragique pour les Romains, et le comique pour eux" (29.4). Ils le compa rent donc à un acteur qui change de masque en fonction du genre de pièce qu'il joue. Et ils ne prennent pas plus au sérieux son masque tragique que son masque comique. Pour eux, Antoine ne cesse pas de jouer, où qu'il se trouve. S'il en est ainsi, à la lecture des nomb reux épisodes où il se déguise et quelles que soient les raisons qui le conduisent à le faire, on peut se demander quelle est la véritable personnalité d'Antoine. Loin d'apparaître comme une entité identifia- 
ble et fermement établie, elle semble incertaine, floue et paraît fluctuer au gré des circonstances et des influences.

Plutarque souligne, en effet, les nom breuses influences qui s'exercent sur Antoine. Elles se succèdent dans sa vie et en déterminent les phases. Il y a d'a bord celle de Curion qui jette Antoine dans une vie de plaisirs et de luxe rui neuse. Plutarque reprend à son compte la tradition selon laquelle cette influence s'abattit sur son personnage "comme un

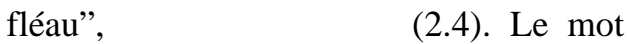
кท่ a un contenu très riche. "Il participe à la fois aux notions de destin, de mort et de démon personnel"' 7 . Plutarque consi dère la fréquentation et l'amitié de Curion comme une malédiction dont Antoine est victime sans l'avoir cherché. Ainsi donc, Antoine, dès sa jeunesse, ne dirige pas sa vie, mais la subit. Il est bal lotté d'une influence à une autre. Après celle de Curion vient celle de Clodius de qui il s'écarte bientôt, par lassitude et par crainte (2.6-7). Il se rapproche ensuite de César à l'instigation de Curion (5.2). Plus tard, il est attiré par le tribun Dolabella qui veut obtenir son soutien à une loi sur une remise de dettes qu'il veut proposer. Cependant, Asinius Pollion et Trebellius poussent Antoine à combattre cette pro position. Ainsi soumis à des influences contradictoires, Antoine finit par s'oppo ser à Dolabella, non pour des raisons politiques, mais parce qu'il le soupçonne d'être l'amant de sa femme (9.1-4). Il épouse ensuite Fulvie qui ne tarde pas à régenter sa vie (10.5-6). Anticipant sur la suite de son récit, Plutarque souligne la dette de Cléopâtre à l'égard de Fulvie : elle lui devait, dit-il, "des gages pour avoir enseigné à Antoine la soumission à l'autorité d'une femme" $\delta ı \delta \alpha \sigma \kappa \alpha \lambda i \alpha .$.

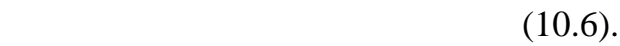
Il établit ainsi une continuité entre les influences exercées par les deux femmes sur Antoine. Cette continuité ne tient pas à la qualité de ces influences, car celle de Fulvie est positive, tandis que celle de Cléopâtre se révélera désastreuse. Elle tient à la soumission d'Antoine qui accepte, dans les deux cas, d'être dirigé par une femme. On a interprété ce ren versement des usages traditionnels, qui voulaient, au contraire, qu'une épouse fût éduquée par son mari, en termes de dévi rilisation et d'émasculation ${ }^{8}$. Envisagée du point de vue du caractère d'Antoine, une telle inversion de la coutume ne fait qu'accroître l'énigme. Un personnage si facile à soumettre et qui se laisse ballotter d'une influence à l'autre possède-t-il vraiment un caractère permettant son identification psychologique ? Plutarque présente sans cesse Antoine comme un homme sous influence. Il lie son identité à la série des tutelles qu'il subit.

Celle de Cléopâtre en constitue le point culminant. Elle inspire à Antoine une passion que Plutarque qualifie de

7 P. Chant raine, 1990, s. Kìjo.

8 B. F. Russel I , 1998. 


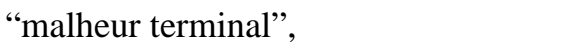
(25.1) . Ces mots expriment à la fois une explication psychologique, un jugement moral et une prophétie post eventum. Cléopâtre est le dernier personnage dont Antoine va subir l'influence, parvenant avec elle au terme d'une vie tout entière tissée d'influences subies. D'autre part, son influence sera néfaste. Enfin, elle sera fatale, car l'adjectif $\tau \varepsilon \lambda \varepsilon v \tau \alpha 10 v$ est dérivé du substantif $\tau \varepsilon \lambda \varepsilon v \tau \eta \dot{~ q u i ~ d e ́ s i ~}$ gne la fin et souvent la fin de la vie ${ }^{9}$. Son amour pour Cléopâtre causera la perte d'Antoine. Pour Plutarque, il agit d'a bord comme un révélateur en réveillant chez son personnage des passions ca chées et en étouffant en lui les restes d'honnêteté qui auraient pu le sauver (25.1) . Cette révélation n'a rien de for tuit. Elle résulte de la détermination de Cléopâtre, bien décidée à séduire An toine avant même de l'avoir rencontré (25.4-5). La reine parvient à ses fins en dirigeant les événements d'un bout à l'autre de leur rencontre (25-27). Elle établit ensuite son autorité sur Antoine en ne le quittant jamais et en le plon geant dans une vie de divertissement perpétuel (28-29). Dès lors, Antoine peut bien s'éloigner d'elle et même ne plus la voir pendant trois ans. L'amour qu'il lui porte, cette "terrible calamité",

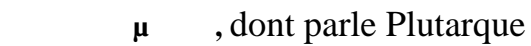

(36.1) demeure. Il lui inspire un com portement aberrant : Antoine engage ainsi prématurément la campagne contre les Parthes parce qu'il est pressé d'aller retrouver Cléopâtre. Plutarque commen te ainsi sa décision : “// $n$ 'était pas diri gé par sa raison, mais sous Vempire de quelques drogues ou sorcellerie, il tour nait toujours ses regards vers elle" (37.6). Antoine n'est donc plus lui-mê me. Sa personnalité disparaît, aliénée par son amour pour la reine. Cet amour prévaut aussi contre les tentatives d'Oc tavie, la seconde épouse d'Antoine, déjouées par les ruses de Cléopâtre qui joue les amantes délaissées et feint de dépérir (53). Et il inspire à Antoine des décisions fatales : celui-ci, pour complai re à Cléopâtre, décide d'affronter Octave sur mer (62.1-2). A Actium, dès qu'il voit Cléopâtre s'enfuir avec ses vaisseaux, il la suit, abandonnant sa flotte en pleine bataille (66.5-8). La manoeuvre de Cléopâtre était peut-être justifiée sur le plan militaire ${ }^{10}$. Plutarque n'en donne aucune explication. Il concentre son récit sur la conduite d'Antoine ${ }^{11}$. Il commente sa désertion en citant une formule de Caton l'Ancien"12 : "L'âme de l'amant vit

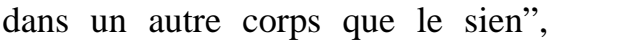

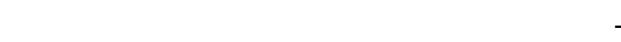
$\alpha \tau 1 £ \dot{\eta} v$ (66.7). Il ne mentionne pas le nom de Caton, sans doute parce que la

9 Voir P. Chant raine, 1990, s. $u$. $\tau \dot{\varepsilon} \lambda$ o .

10 Voir H. Beng st o n, 1977, 243-245.

11 F. E. Brenk, 1992,4445.

12 Voir C. Mai 9, 8. Amatorius 759c. 
présence du nom d'un illustre Romain serait déplacée dans un épisode où An toine oublie les vertus romaines, et en particulier le courage guerrier ${ }^{13}$, mais aussi peut-être parce qu'elle aurait pu distraire l'attention du lecteur de l'es sentiel, à savoir le comportement aliéné d'Antoine. Plutarque le décrit ainsi :

Dans cette circonstance, Antoine montra qu'il se dirigeait lui-même sans suivre les raison nements d'un chef, ni d'un homme ni, en un mot, les siens propres... entraîné qu'il était par cette femme comme s'il ne faisait qu'un avec elle et qu'il était emporté avec elle (66. 7).

Plutarque avait déjà souligné que la passion d'Antoine pour Cléopâtre était placée sous le signe de l'aliénation. Celle-ci atteint son point culminant pen dant la bataille d'Actium. Au moment où Antoine abandonne le combat, il n'est plus personne, ni un chef, ni un homme, ni lui-même, il est devenu une partie de Cléopâtre ( $\sigma \cup \pi \beta \varphi \cup \kappa \dot{\omega}$ ), ce qui explique qu'il soit emporté dans la même mouvement qu'elle ( $\sigma v{ }^{\wedge} \tau \alpha \varphi \mathrm{i} \Xi$ -

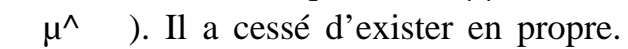
Son aliénation complète fait disparaître son caractère, puisqu'il n'agit plus qu'en fonction des actions de la reine. Il lui est entièrement asservi. C'est ainsi que la propagande octavienne le représentera. Mais Plutarque, tout en utilisant cette tradition, se garde de toute polémique politique. Il adopte une point de vue moral ${ }^{14}$. Il relève chez Antoine une vacuité intérieure qui pose à nouveau la question de sa personnalité. Celle-ci existe-t-elle vraiment ? Après son éclip se au début de la campagne contre les Parthes, Plutarque met à nouveau en relief une autre de ses disparitions à un moment bien plus crucial. N'est-elle donc qu'une absence qui, par les consé quences qu'elle entraîne, prend la forme d'une sorte de grandeur négative ? Les derniers temps de la vie d'Antoine invi tent encore à s'interroger à ce sujet.

Après Actium, Antoine revient en Egypte, mais quitte Alexandrie. Il s'iso le dans une maison au bord de la mer (69.6), construite sur une jetée. Il y vit "en exilé loin des hommes", puyá $\alpha v$ $\theta \rho \dot{\omega} \pi \omega v$ (69.7), en prenant Timon d'Athènes pour modèle :

Il déclarait qu'il aimait et vou lait imiter la vie de Timon, parce qu'il avait connu un sort analogue au sien. Lui aussi, en effet, victime de l'injustice et de l'ingratitude de ses amis, éprouvait pour cette rai son de la défiance et de la haine à l'égard de tous les hommes (69.7).

Si cet épisode dure peu, puisqu'An toine regagne bientôt Alexandrie pour y reprendre, avec Cléopâtre, une vie de fê tes en attendant l'arrivée d'Octave et la mort (71.2-3), il n'en est pas moins frap-
C. B. R. Pell in g, 1988, 285.
14 F. Cha moux, 1986, 389-393. 
pant. Il présente d'abord un caractère paradoxal : Antoine, le chef aimé de ses soldats et des foules d'Asie et d'Egypte, "le dernier prince de l'Orient grec"15, devient un solitaire misanthrope. Pour cette nouvelle phase de sa vie, il se choi sit un modèle, à première vue le plus étranger qui soit à sa manière de vivre flamboyante, Timon d'Athènes, dont Plutarque rapporte certains traits pour instruire le lecteur (70). Celui-ci, ainsi éclairé sur la haine que cet Athénien du Vè siècle av. J. C. vouait à ses compa triotes, ne peut manquer d'être frappé par le changement d'Antoine qui pré tend suivre un pareil guide. Mais il y a plus surprenant encore : alors même que sa carrière politique est achevée et que son destin est scellé, Antoine a encore besoin, pour parler de lui, de se référer à quelqu'un d'autre. Sa vie a été modelée par Curion, par Clodius, par Fulvie, par Cléopâtre. Elle va maintenant l'être par le souvenir de Timon. Une fois de plus, le caractère d'Antoine n'existe que comme reflet d'une influence extérieure. Antoine a été un fêtard comme Curion et Clodius, un époux docile, comme le sou haitait Fulvie, un amant passionné et soumis comme le voulait Cléopâtre. Il va maintenant devenir un misanthrope à l'image de Timon. Et la brièveté de sa conversion à la misanthropie illustre une fois de plus son instabilité psycholo gique qui paraît liée à une absence de caractère propice à tous les errements.

Loin de dissimuler cette instabilité, Plutarque la met en valeur et cherche à l'expliquer. Il note qu'Antoine avait un caractère simple et qu'il était lent à s'a percevoir de la vérité, mais prompt à reconnaître ses fautes et à s'en repentir (24.10). Il le présente surtout comme la victime des flatteurs qui l'entouraient. Ces derniers, en montrant de la franchi se lorsqu'ils se moquaient de lui, parve naient à lui faire croire qu'il disaient la vérité lorsqu'ils lui parlaient sérieuse ment. Ils exerçaient ainsi sur lui une influence néfaste (24.12). C'est une ana lyse ingénieuse mais qui, comme l'a bien vu C. B. R. Pelling ${ }^{16}$, manque de bases historiques. Si Plutarque évoque l'indignation suscitée chez les honnêtes gens par la vie déréglée que menaient Antoine et son entourage ${ }^{17}$, il ne relate aucun cas particulier de flatterie perni cieuse et ne désigne aucun flatteur par son nom. Lorsqu'il parle de la flatterie de Cléopâtre $(29,1)$, il désigne par ce terme l'ensemble des artifices que la reine déploie pour procurer sans cesse à Antoine des plaisirs nouveaux et pour le garder ainsi sous sa coupe. Mais c'est à un tout autre type de flatterie, la flatterie de cour, qu'il attribue les erreurs d'Antoine, sans en citer d'exemples pré cis $(24,12)$. En fait, il applique alors à

15 F. Ch a mo ux, 1986.

16 C. B. R. Pell ing, 1988, 182.

$17 \quad 9.5-9.24 .2-5$ 
son cas le schéma de la réflexion qu'il développe dans le traité intitulé Com ment distinguer le flatteur de l'ami.

Ce traité ne peut être daté avec pré cision. Il appartient probablement aux oeuvres composées par Plutarque pen dant sa maturité ${ }^{18}$. Il est dédié à Antiochos Philopappos, dernier roi de Commagène, et se présente comme un manuel de morale pratique censé per mettre à ce souverain de distinguer dans son entourage les flatteurs et les amis sincères. En réalité, ce sujet n'est traité que dans la première partie (1-24), où Plutarque expose les critères et analyse les indices qui peuvent permettre d'opé rer la distinction annoncée dans le titre. La seconde partie (25-37) est consacrée au bon usage de la franchise entre amis. Mais la franchise ne se rencontre pas seulement dans les conversations sincè res. Plutarque soutient, en effet, que le flatteur excelle à contrefaire le compor tement des vrais amis et qu'il y parvient, entre autres, en imitant leur franchise :

Mais ce qui est le plus pervers de tout chez lui, c'est que, se ren dant compte que la franchise, à ce qu'on dit et à ce qu'il semble, est la voix propre à l'amitié, comme un animal en a une propre, tandis que le manque de franchise est étranger à l'amitié et étranger à la noblesse, il ne la laisse pas elle non plus à l'écart de ses imita tions mais, comme les cuisiniers habiles ont recours aux saveurs amères et aux condiments âcres pour faire disparaître le dégoût que peuvent susciter les aliments sucrés, de même les flatteurs emploient une franchise qui n'est ni authentique ni utile mais qui, pour ainsi dire, fait les gros yeux en fronçant les sourcils et se borne à chatouiller $(51 \mathrm{c}-\mathrm{d})$.

Plutarque reprend, dans la Vie d'An toine, la même idée et presque les mêmes mots pour expliquer l'influence des flat teurs sur son personnage. Celui-ci ignorait "que certains mêlaient la franchise comme un condiment un peu âpre à leur flatterie pour faire disparaître le dégoût qu'elle pouvait susciter" (24.12). Plutarque ap plique donc au cas d'Antoine sa théorie relative à l'utilisation de la franchise par les flatteurs. Quel que soit l'ordre de com position de ses deux oeuvres, elles illust rent toutes les deux la constance et la cohé rence de sa pensée. Mais les coïncidences entre elles ne s'arrêtent pas là.

Dans le traité, Plutarque se réfère en effet à deux reprises au cas d'Antoine. Soulignant qu'un des pires effets de la flatterie est de faire passer les vices pour des vertus et d'amener celui qui en est affligé à s'y complaire, il affirme que c'est ce phénomène "qui a presque sub verti et détruit la puissance des Romains qui était alors si grande en désignant par des expressions atténuées les débauches, les dérèglements et les exhibitions 
d'Antoine comme des comportements pleins de gaieté et d'humanité dûs au pouvoir et à la fortune qui le traitaient généreusement" (56e). Plus loin, Plutar que décrit une autre ruse des flatteurs : ils blâment parfois à dessein des attitu des qui sont à l'opposé de celles qu'a dopte la personne qu'ils flattent. Ils l'en couragent ainsi à persévérer. Pour illust rer son propos, il recourt de nouveau à l'exemple d'Antoine :

C'est ainsi que les amis d'Antoine, qui était amoureux de Cléopâtre et qui brûlait pour elle, le persuadaient qu' elle était amoureu se de lui et, lui faisant des repro ches, le traitaient d'insensible et d'orgueilleux. "Cette femme", dis aient-ils "a abandonné un si grand royaume et un genre de vie heureux pour se consumer à participer à des campagnes à tes côtés, en tenue de concubine, mais dans ta poitrine, il $y$ aun coeur insensible aux enchan tements.." Et lui, prenant plaisir à être critiqué pour ces fautes et tirant de ses accusateurs une joie que ne lui procuraient même pas ceux qui faisaient son éloge était à son insu perverti davantage par cette appa rente réprimande (61a-b).

Dans ce traité comme dans la Vie qu'il lui consacre, Plutarque présente donc Antoine comme une victime des flatteurs. Et son jugement suscite les mêmes questions sur le caractère de son personnage. Elles prennent même un relief particulier si on les rapproche de la perspective qu'il adopte pour réfléchir à la flatterie.
Il distingue d'abord les parasites des flatteurs. Le but et la bassesse des premiers sont faciles à reconnaître (3). Les seconds, en revanche, s'avancent masqués et l'on doit donc être en garde contre eux (4). Quel est leur but ? Plutarque ne l'indique pas. On se doute, bien sûr, qu'ils trouvent leur intérêt à flatter, mais Plutarque n'ex plique pas ce qu'ils y gagnent. Il ne se place pas dans une optique utilitaire. Il envisage la flatterie d'un point de vue psychologique et moral. Il la décrit comme une situation existentielle où un homme s'efforce de donner à un autre homme une fausse image de lui-même. Tel est le dan ger principal couru par l'homme que l'on flatte : il perd la connaissance de luimême. Aussi le flatteur s'avère-t-il l'enne mi de l'Apollon Pythien puisqu'il contra rie la mise en oeuvre de la maxime delphienne "Connais-toi toi-même" (49a-b). Il prend pour cible le caractère de celui qu'il flatte et finit par altérer sa nature, lui causant ainsi un dommage très grave :

Ceux qui parviennent jusqu'au caractère par leurs éloges et, par Zeus, atteignent la manière de pen ser par leur flatterie font comme les esclaves qui volent en se servant non dans le tas de grain, mais dans la semence. En effet, alors que les dispositions intérieures et le carac tère sont la semence des actions, ils leur infligent des distorsions en tant que principe et source de la vie en attribuant au vice les noms de la vertu (56b).

Le flatteur finit ainsi par dénaturer le caractère de celui qu'il flatte. Il le fait 
devenir quelqu'un d'autre. La flatterie se révèle donc une entreprise d'aliénation. Dans cette entreprise, le flatteur a pour allié l'amour-propre de sa victime (48e-49a), mais aussi sa propre plasticité. En effet, il n'est rien en lui-même. Il n'existe qu'en adoptant la forme de celui qu'il flatte :

Le flatteur, parce qu'il n'a pas un foyer unique et stable pour son caractère et qu'il ne vit pas une vie qu'il a choisie pour lui-même, mais pour un autre, et qu'il se modèle sur un autre et s'adapte à lui, n'est pas simple ni unique, mais varié et changeant, tombant sans cesse d'une forme dans une autre comme l'eau qu'on transvase et changeant d'apparence en fonction de ceux qui le reçoivent. (52a-b)

Le flatteur est donc lui aussi aliéné. Il n'a pas de caractère propre et stable. Il ne vit pas pour lui, mais pour un autre. Il ne choisit pas la forme de sa vie, mais la modèle sur celle d'un autre et change en fonction des cibles qu'il a choisies. Cependant, il ne subit pas ces change ments, il les organise. Son aliénation est volontaire et consiste dans une dyna mique d'adaptation permanente, tandis que celle de ses victimes leur est infligée par lui. Plutarque décrit donc la flatterie comme un affrontement étrange entre un caractère menacé de dénaturation par le flatteur qui lui tend des pièges et un autre caractère, celui du flatteur, dépourvu de nature propre et qui épouse la forme du caractère qu'il veut dénaturer. Dans cet affrontement n'existe donc ni d'un côté ni de l'autre de caractère dont l'identité soit clairement définie et la stabilité garantie. On y retrouve ainsi la même incertitude relative au caractère qui marque le cas d'Antoine dans la Vie d'Antoine. Aussi Plutarque se réfère-t-il deux fois à ce cas pour illustrer cette incertitude dans son traité. Ce cas n'est pas le seul exemple cité dans ce texte, mais sa présence éclaire et confirme sa nature énigmatique qui appa raissait dans la Vie d'Antoine.

En racontant sa vie, Plutarque décrit Antoine comme un homme incertain qui passe d'un vêtement à un autre, d'une influence à une autre et qui est la proie des flatteurs. En définissant, dans le trai té Comment distinguer le flatteur de l'ami, l'activité des flatteurs comme la conjonction de deux aliénations dont l'une provoque l'autre, Plutarque cite aussi Antoine comme exemple d'un homme aliéné par les flatteurs qui l'en touraient. Il pose ainsi à deux reprises la question du caractère d'Antoine. Il dit avoir voulu raconter sa vie et celle de Démétrios comme un contre-exemple moral destiné à accroître, par réaction, le désir d'imiter les vies des hommes ver tueux ${ }^{19}$. Mais la clarté de ce programme ne se retrouve pas dans sa réalisation. $\mathrm{La}$ Vie d'Antoine est placée sous le signe de la complexité psychologique, parce que la nature et l'existence même du carac tère d'Antoine y apparaissent comme problématiques. Plutarque ne cherche

19 Dtr. 1. 
pas à dissiper artificiellement l'obscurité qui enveloppe l'objet qu'il veut repré senter, il la met au contraire en relief. Ainsi se montre-t-il sans doute un peint re fidèle en même temps qu'il révèle les incertitudes propres à l'exploration qui est au coeur de l'écriture des Vies.

Bibl iographie

Bengst on, H.,

- Marcus Aurelius Triumvir und Herrscher- Plutarch. Life of Antony, Cambridge, 1988. des Orient, Münich, 1977.

Brenk, F. E.,

- "Plutarch's Life « Markos Antonios » : A Literary and Cultural Study", ANRW II.
33. 6(1999), 4347-4469.

Cha moux, F.,

- Marc Antoine dernier prince de T Orient grec, Paris, 1986.

Chant raine, $R$,

- Dictionnaire étymologique de la langue grecque, Paris, rééd. 1990.

Ga 1 l o, I. \& Pe t t ine, E. (eds.),

- Plutarco. Come distinguere Tadulatore dall amico, Naples, 1988.

Pelling, C. B. R.

Russelı, B. F,

- "The Emasculation of Antony: The Construction of Gender in Plutarch's Life of Antony”, Helios, 25 (1988) 121-137. 